\title{
Manipulation of phytobiome: a new concept to control the plant disease and improve the productivity
}

\begin{abstract}
Various strategies are currently being developed to improve sustainable agricultural production. Many concepts at present in market to control the plant disease. Most widely used were chemicals or pesticides. Commercial biological control products for the use against plant diseases must be highly efficient against the targeted disease. Second concept to control the disease through bio-agents. Many bio-agents available in market but not effective as chemical agents so not popularized as compare to chemical agents. Microbes more diversity found in rhizosphere so rhizosphere is called house of microbes. Interaction of microbes in rhizosphere will decide the health of plant. It is now widely recognised that plant micro biota provide important functions for their host's performance, and mediate functions like nutrient delivery, fitness, stress tolerance, and pathogen or pest control. Current understanding of plantmicrobe interactions is helping to develop microbial products, new applications to improve crop production, and create alternatives to chemicals. Two types of microbes present in rhizosphere culturable and non-culturable. Plant health not depend on only culturable microbes but also depend on non-culturable microbes. it is necessary to study non-culturable microbes. Today new era of microbiology is metagenomics. It is possible to development of various sequencing technologies specific to next generation sequencer. Metagenomics means to study of culturable as unculturable microbe. Manipulation of microbial population in rhizosphere directly affect the plant health and productivity of plant. This strategies will be used for control the plant disease.
\end{abstract}

Keywords: phytobiome, rhizosphere, microbial population, microorganisms
Volume 6 Issue 6 - 2018

Manoj V Parakhia, Golakiya BA

Department of Biotechnology, Junagadh Agricultural University, India

Correspondence: Manoj V Parakhia, Department of Biotechnology, Junagadh Agricultural University, Junagadh, Gujarat, India, Email mvparakhea@gmail.com

Received: June II, 20I8 | Published: November 23, 2018

\section{Introduction}

Now day we face an unprecedented challenge to feed the growing global human population. To overcome the challenge by with major changes in how we combine science with agronomy. The green revolution is thought to have saved approximately 1 billion lives and was largely fuelled by effective plant genetic improvement (breeding) programs. All plants live with microbes and it is well known that many rhizosphere microbes can be beneficial for plant growth. However, microbiologists have largely ignored the principals used by plant breeders of taking naturally occurring genetic variation as a base to genetically improve microorganisms to increase crop production.

\section{Effect of microbes on plant}

Microbes has received substantial attention in recent years. ${ }^{1,2}$ A testament to the importance of plant-microbe interactions are the mycorrhizal fungi. Molecular evidence suggests that their associations with green algae were fundamental to the evolution of land plants about 700 million years ago. ${ }^{3}$ Most plants, although notably not Arabidopsis thaliana and other Brassicaceae, have maintained this symbiosis, which assists root uptake of mineral nutrients such as phosphate. ${ }^{4}$ Plant-associated microbes are also key players in global biogeochemical cycles. ${ }^{5}$ A significant amount, 5 to $20 \%$, of the products of photosynthesis (the photosynthate) is released, mainly into the rhizosphere (the soil-root interface) through roots. ${ }^{6}$ In addition, $100 \mathrm{Tg}$ of methanol and $500 \mathrm{Tg}$ of isoprene are released into the atmosphere by plants annually., ${ }^{7,8}$ For methanol this corresponds to between $0.016 \%$ and $0.14 \%$ of photosynthate depending on plant type. ${ }^{9}$ Both are potential sources of carbon and energy for microorganisms. In agricultural soils in particular, plants stimulate microbial denitrification and methanogenesis, which contribute to emissions of $\mathrm{N}_{2} \mathrm{O}$ and methane, respectively. ${ }^{10,11}$ These gases represent a loss of carbon and nitrogen from the system and contribute to the greenhouse effect.

Manipulation of the plant microbiome has the potential to reduce the incidence of plant disease. ${ }^{12,13}$ increase agricultural production, ${ }^{14}$ reduce chemical inputs, ${ }^{15}$ resulting in more sustainable agricultural practices. This goal is seen as vital for sustaining the world's growing population.

The plant microbiome is a key determinant of plant health and productivity. ${ }^{16}$ Phytobiome of plants are known for their importance for healthy growth and development of the host. Similar to modern therapeutic concepts in human and veterinary medicine modifications of plant microbiomes will be an essential component of tomorrow's crop production and protection. It will also allow for making use of the full potential of naturally occurring microbes and microbial functions. Phytobiome modulation is a new approach, which currently receives extremely high attention by science and industry. However, targeting the plant microbiome for improved crop production requires profound knowledge on plant microbiome functioning and dynamics. To apply (meta) genomic approaches to studying the bacterial community compositions and dynamics in different crops and model plants and modern transcriptomics to elucidate the genetic basis of beneficial plant-microbe interaction and communication mechanisms.

Phytobiome study proposes to examine how the microorganisms associated with plants, the plant microbiome can be engineered to 
improve crop quality and yield using synthetic biology approaches. to manipulation of rhizosphere microbiome population for the control of plant disease. Here manipulation means the change population of specific microbes species.

Microbes affect the plant health by various way by antagonist to the pathogen microbes and affect the growth of pathogenic organisms. Plant secret the metabolite which act as growth stimulators for the organisms live in rhizosphere. The studied of rhizospheric microorganisms suggested that the composition on microorganisms of stress plant vs healthy plant was different. Stress may any type biotic or abiotic. Microbes are of two types culturebal as well as Non-culturebal. microbes which are culturebal documented well but needs to more study on non-culturebal microbes because the effect of microbes on plant that we observed was due to not only culturebal microorganisms but also due to combination with non-culturebal microbes.

\section{Pathogenic microorganisms}

Many bacteria, fungi and viruses were caused the diseases in many plants and identified as phytopathogenic microorganisms, due to pathogenic microorganisms every year yield losses worldwide. This is the main problems for farmer.

Second type of microorganisms help to plant for growth and development are called beneficial microorganisms. Microbes help to plant by providing nutrients from the soil. Some microbes fixed the environmental nitrogen and provide to the plant. Some microbes present in soil that convert insoluble compounds to soluble compounds. Specifically Microbes which are present around the area nearer to root zone, this zone is called rhizosphere. Various environmental factors affect the plant like temperature, salt drought etc. These factors are called abiotic stress factors. Living organisms caused the disease called biotic factors.

Rhizosphere is the factories of microorganisms because most diversity of microbes found in rhizosphere ecosystem than other ecosystem. Plant health depended on the rhizosphere of its root zone. Manipulation of rhizospheric microorganisms will affect the overall impact on plant growth and crop production. Manipulation in the sense of change the composition of microorganisms means the increase the no. of beneficial microbes like siderophore producing, phosphorus solubilising, zinc solubilising, Nitrogen fixing bacteria etc. Which affect the growth of harmful organisms, which overall increase the plant growth and crop production.

Technologies or methods used for crop improvement in breeding was marker assistant selection programme. same the technologies will be developed using the newly techniques as microbes assisted crop production.

\section{Strategies for study of non-culturabal microbes}

Study of Phytobiome require the DNA sequencer, last 10-12 years the metagenomics shotgun sequencing transfer to Sanger sequencing technology to Next-generation sequencing. The Sanger sequencing technologies is still consider gold standard for sequencing. due to the process, overall cost and output data of sanger sequencing approach shifted to Next generation sequencing technologies. NGS have low cost, process simple and large data output. NGS like 454/Roche, Illumina/Solexa, Ion-torrent, Ion-S5, Pac bio sequencer were used to sequencing of metagenomics's samples. Following flow chart reperesent the over all process of phytobiome study.
Samples: Soil sample, Root sample, leaf sample, waste sample<smiles>[CH]1[CH]C=C1</smiles>

Direct DNA extract from the above samples using available Kits or manual protocol.<smiles>[C+]1[C+]=CCC1</smiles>

Purification of Extracted DNA<smiles>[CH]1C=C1</smiles>

Library preparation form purified DNA according to NGS platform and manufacture instructions. (Using specific primers)<smiles>[CH]1CC1</smiles>

Library will be sequenced using Next generation sequencer.

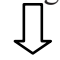

NGS data analysed using various bioinformatics' software.<smiles></smiles>

Identified the Operation Taxonomic Unit (OTU)<smiles>[C+]1=CC=C1</smiles>

Comparison of Data with control and identified dominant specie. (Identified species will be used against the biotic and abiotic stresses)

\section{Conclusion}

Rhizosphere is most divers than other ecosystem of microbes. Microbes interaction with plant in rhizoshere will decide to the plant health. Yet to date so much studied completed on rhizospheric microbes but only study culturable microbes. It was identified that the 1-2 \% of microbes were culturable while $98-99 \%$ microbes were non-culturable, interaction in rhizosphere with plant participated both types of microbes culturable as well as non-culturable. So now attention require to study the non-culturable microbes and its effect on the plant. The development of sequencing technologies it is now possible to study non-culturable microbes. Development of high through put next generation sequencing technologies. That easy and cost effective for the study of un-culturable microbes, the new era was developed that metagenomics, in this DNA directly extracted for soil and prepare library for sequencing after that carry out the sequencing.

Manipulation of rhizospheric microbes population means the changes of specific species population in rhizosphere would affect the plant. Due to phytobiome study it is possible to studied whole population of microbes in the rhizosphere based on the microbes population in the rhizosphere of healthy plant Vs disease (stress) plant identified the dominant species in the rhizosphere's of stress plant, this identified dominant species will be used for the control of disease. Using this concept many studied were completed and that was success to control the disease in field condition. So now more attention will be given to study of microbiome of rhizosphere.

\section{Acknowledgments}

We thank to professor and head, department of biotechnology, JAU, Junagadh that give me opportunity to write this review on phytobiome.

\section{Conflicts of interest}

The author declares there is no conflicts of interest.

\section{References}

1. Lebeis SL, Rott M, Dangl JL, et al. Culturing a plant microbiome community at the cross-Rhodes. New Phytol. 2012;196(2):341-344. 
2. Bulgarelli D, Schlaeppi K, Spaepen S, et al. Structure and functions of the bacterial microbiota of plants. Annu Rev Plant Biol. 2013;64:807-838.

3. Heckman DS, Geiser DM, Eidell BR, et al. Molecular evidence for the early colonization of land by fungi and plants. Science. 2001;293(5532):1129-1133.

4. Bonfante P. Plant-fungal interactions in mycorrhizas. eLS. 2010.

5. Philippot L, Hallin S, Borjesson G, et al. Biochemical cycling in the rhizosphere having an impact on global change. Plant Soil. 2009;321(1-2):61-81.

6. Marschner. Mineral Nutrition in Higher Plants. Academic Press, London; 1995.

7. Galbally IE, Kirstine W. The production of methanol by flowering plants and the global cycle of methanol. J Atmos Chem. 2002;43(3):195-229.

8. Wang KY, Shallcross DE: Modelling terrestrial biogenic isoprene fluxes and their potential impact on global chemical species using a coupled LSM-CTM model. Atmos Environ. 2000;34(18):2909-2925.

9. Wrage N, Velthof GL, van Beusichem ML, et al. Role of nitrifier denitrification in the production of nitrous oxide. Soil Biol Biochem. 2001;33(12-13):1723-1732.
10. Conrad R, Erkel C, Liesack W. Rice Cluster I methanogens, an important group of Archaea producing greenhouse gas in soil. Curr Opin Biotechnol. 2006;17(3):262-267.

11. Bloemberg GV, Lugtenberg BJJ. Molecular basis of plant growth promotion and biocontrol by rhizobacteria. Curr Opin Plant Biol. 2001;4(4):343-350.

12. Andrews JH. Biological-control in the phyllosphere. Annu Rev Phytopathol. 1992;30:603-635.

13. Bakker MG, Manter DK, Sheflin AM, et al. Harnessing the rhizosphere microbiome through plant breeding and agricultural management. Plant Soil. 2012;360(1-2):1-13.

14. Adesemoye AO, Torbert HA, Kloepper JW. Plant growth-promoting rhizobacteria allow reduced application rates of chemical fertilizers. Microb Ecol. 2009;58(4):921-929.

15. Singh BK, Bardgett RD, Smith $\mathrm{P}$, et al. Microorganisms and climate change: terrestrial feedbacks and mitigation options. Nat Rev Microbiol. 2010;8(11):779-790.

16. Berendsen RL, Pieterse CMJ, Bakker P. The rhizosphere microbiome and plant health. Trends Plant Sci. 2012;17(8):478-486. 